\title{
Associations between the human intestinal microbiota, Lactobacillus rhamnosus GG and serum lipids indicated by integrated analysis of high-throughput profiling data
}

Accumulating evidence indicates that the intestinal microbiota regulates our physiology and metabolism. Bacteria marketed as probiotics confer health benefits that may arise from their ability to affect the microbiota. Here high-throughput screening of the intestinal microbiota was carried out and integrated with serum lipidomic profiling data to study the impact of probiotic intervention on the intestinal ecosystem and on host, and to explore the associations between the intestinal bacteria and serum lipids. We performed a comprehensive intestinal microbiota analysis using a phylogenetic microarray before and after Lactobacillus rhamnosus GG intervention. While a specific increase in the L. rhamnosus-related bacteria was observed during the intervention, no other changes in the composition or stability of the microbiota were detected. After the intervention, lactobacilli returned to their initial levels. As previously reported, also the serum lipid profiles remained unaltered during the intervention. The most prevailing association between the gut microbiota and lipid profiles was a strong positive correlation between uncultured phylotypes of Ruminococcus gnavus-group and polyunsaturated serum triglycerides of dietary origin. Moreover, a positive correlation was detected between serum cholesterol and Collinsella (Coriobacteriaceae). These associations identified with the spectrometric lipidome profiling were corroborated by enzymatically determined cholesterol and triglyceride levels. Actinomycetaceae correlated negatively with triglycerides of highly unsaturated fatty acids while a set of Proteobacteria showed negative correlation with ether phosphatidylcholines. Based on a high-resolution microbiota analysis, intake of L. rhamnosus GG did not modify the composition of the intestinal ecosystem in healthy adults, indicating that probiotics confer their health effects by other mechanisms. Our results suggest that several members of the Firmicutes, Actinobacteria and Proteobacteria may be involved in the metabolism of dietary and endogenous lipids, and provide a scientific rationale for further human studies to explore the role of intestinal microbes in host lipid metabolism. 
1 Leo Lahti*, Department of Veterinary Bioscience, University of Helsinki, Finland; Laboratory of

2 Microbiology, Wageningen University, Netherlands

3 Anne Salonen*, Department of Veterinary Bioscience, University of Helsinki, Finland; Department of

4 Bacteriology and Immunology, University of Helsinki, Finland

5 Riina A. Kekkonen, Valio R\&D, Helsinki, Finland

6 Jarkko Salojärvi, Department of Veterinary Bioscience, University of Helsinki, Finland

7 Jonna Jalanka-Tuovinen, Department of Veterinary Bioscience, University of Helsinki, Finland

8 Airi Palva, Department of Veterinary Bioscience, University of Helsinki, Finland

9 Matej Orešič, VTT Technical Research Centre of Finland, Espoo, Finland

10 Willem M. de Vos, Department of Veterinary Bioscience, University of Helsinki, Finland; Laboratory

11 of Microbiology, Wageningen University, Wageningen, Netherlands; Department of Bacteriology and

12 Immunology, University of Helsinki, Finland

13

14 *equal contribution;

15

16 Corresponding author:

17 Name: Leo Lahti

18 Address: Dreijenplein 10, Wageningen University, 6703HB Wageningen, Netherlands

19 Tel: +31 616737991

20 E-mail: leo.lahti@iki.fi 


\section{Introduction}

22 Over $90 \%$ of the cells in the human body constitute microbes, the majority of them living in the lower

23 part of the gastrointestinal (GI) tract. Hence, we are composite organisms programmed not only by the

24 inherited and stable human genome but also by the environmentally acquired and plastic microbiome.

25 The coding capacity of the microbiome vastly surpasses the human genome with more than three

26 million genes (Qin et al. 2010). While many of the microbial functions have not yet been characterized, various mechanisms have been described by which the intestinal microbes impact our life, including

28 the metabolism of our food, exclusion of pathogens and many signaling functions that range from

29 modulation of the mucosal immune response to development of metabolic diseases (Holmes et al.

30 2011; Sekirov 2010). Bacterial metabolism of dietary and endogenous substances is known to generate

31 a wide repertoire of metabolites that may have beneficial or harmful effects on the host (O'Keefe 2008;

32 Blaut \& Clavel 2007). Hence, the intestinal microbiota has a remarkable potential to influence the

33 physiology and biology of the host (Tremaroli \& Bäckhed 2012), and unlike the human genome, its

34 gene pool can be modulated by changing the environmental conditions, such as food or drug intake,

35 affecting the composition and function of the intestinal microbiota (Zoetendal, Rajilić-Stojanović \& de

36 Vos 2008).

37 Consumption of lactic acid bacteria marketed as probiotics is a common approach to maintain

38 health (Saxelin et al. 2005). Lactobacillus rhamnosus GG is one of the most widely used probiotic

39 bacteria that is assumed to interact with the host via binding to human mucus via its extracellular pili

40 (Kankainen et al. 2009). However, the further molecular details of the probiotic signaling are not yet

41 understood and it remains to be established whether the effect is direct, through metabolites or

42 structural components modulating for instance the immune responses of the host or indirect, via

43 alteration of the intestinal microbiota. From the ecological perspective, a single bacterial strain is not

44 likely to radically alter the established intestinal community, which in adults typically consists of 
45 hundreds of different species-level phylotypes that represent approximately ten bacterial phyla, vastly 46 dominated by Firmicutes, Bacteroidetes and Actinobacteria, followed by Proteobacteria, Fusobacteria 47 and Verrucomicrobia (Zoetendal, Rajilić-Stojanović \& de Vos 2008). Many of the currently 48 characterized phylotypes are strict anaerobes, have not yet been cultured, and can only be recognized 49 based on molecular methods (Zoetendal, Rajilić-Stojanović \& de Vos 2008). The development of 50 culture-independent molecular techniques has provided insights in the composition of the intestinal

51 microbiota before and following probiotic intake. Targeted microbiota analyses as well as the more 52 recent community-level profiling studies support the view that the effects of probiotic intake are limited 53 and only affect bacteria related to the probiotics (Satokari et al. 2001; Vaughan, Mollet \& deVos 1999;

54 Palaria, Johnson-Kanda \& O'Sullivan 2011; McNulty et al. 2011).

Animal and in vitro studies have shown that the intestinal microbiota can regulate host lipid metabolism via numerous microbial activities (Fava 2006; Martin et al. 2007). The best characterized mechanism is through the biotransformation of bile acids, which regulate the digestion and absorption 58 of fats, and profoundly affects the cholesterol and other lipid metabolism in the body (Gérard P. 2010; 59 Ridlon, Kang \& Hylemon 2006). However, global monitoring of the serum and organ lipid profiles of 60 germ-free and conventionally raised mice suggests an even more widespread and profound influence of 61 the intestinal microbiota on host lipid metabolism, in particular on triglycerides and 62 phosphatidylcholines (Velagapudi et al. 2010; Orešič, Hänninen \& Vidal-Puig 2008). In the present study, we characterized the impact of a probiotic intervention on the composition 64 and stability of the intestinal microbiota and serum lipid profiles in healthy adults. A comprehensive 65 intestinal microbiota profiling and phylogenetic analysis was carried out with the Human Intestinal 66 Tract Chip (HITChip), a phylogenetic microarray that provides a robust and sensitive measurement 67 platform to assessing the abundance of over 1000 microbial species-like phylotypes representing the 
68 majority of the known bacterial diversity of the human intestinal tract (Rajilić-Stojanović et al. 2009).

69 Stability of the microbiota was quantified by inter- and intra-individual correlations within and between

70 time points. The study subjects were sampled prior and after three weeks consumption of dairy milks

71 supplemented with either the probiotic L. rhamnosus GG or a placebo. Moreover, the microbiota

72 composition was analyzed from additional follow-up samples collected three weeks after the

73 intervention trial. With phylogenetic microarray analysis we could both deepen and expand the typical

74 bacterial analysis in probiotic trials, often limited to specific bacterial groups, such as lactic acid

75 bacteria, or to the dominant fraction of the microbiota. Previously, we have shown that the intake of $L$.

76 rhamnosus GG did not elicit any significant changes in the serum lipids (Kekkonen et al. 2008b). The

77 present study complements these results by providing longitudinal data and evidence that the probiotic

78 intervention with L. rhamnosus GG does not affect the overall composition or stability of the intestinal

79 microbiota. Furthermore, we have integrated the lipid profiling and global microbiota data sets to

80 investigate the overall associations between the intestinal microbes and systemic metabolites, in

81 particular serum lipids. Quantitative analyses suggest that the abundance of specific intestinal microbes

82 is correlated to that of specific lipid species. We identified mainly uncultured members of the

83 Firmicutes, Actinobacteria and Proteobacteria that seem to be involved in the absorption and

84 metabolism of the dietary and endogenous lipids, providing a scientific rationale for further human

85 studies designed to explore the role of intestinal microbes in lipid metabolism, and associations to

86 health.

87

88 Materials and Methods

89 Ethics statement

90 The trial and its protocol have been approved by the Ethics Committee of the Hospital District of

91 Helsinki and Uusimaa (Ethical protocol no HUS 3577E0/05). The subjects provided written informed 
92 consent. The details of this trial have been published previously (Kekkonen et al. 2008a; Kekkonen et 93 al. 2008b).

95 Subjects

96 The subjects were healthy Finnish adults ( $n=25,18$ females, 7 males) with a mean age of 42 years

97 (23-55) and a mean BMI of $24 \mathrm{~kg} / \mathrm{m}^{2}$ (18-30) from Helsinki region, representing a subset of a larger cohort (Kekkonen et al. 2008a; Kekkonen et al. 2008b). The subject characteristics are provided in 99 Supplementary Table S1.

100

\section{Dietary Intervention}

\section{Fecal samples and microbiota analysis with the HITChip and quantitative PCR}

112 Three fecal samples per individual were collected three weeks before, during and three weeks after the 113 intervention, as previously described (Kekkonen et al. 2008b). The fecal DNA extraction with modified 114 Promega method, and quantification of the probiotic counts with a strain-specific quantitative PCR 115 (qPCR) assay have been described previously (Ahlroos \& Tynkkynen 2009). The phylogenetic analysis 
116 of the intestinal microbiota composition with the HITChip microarray was performed as previously

117 described (Rajilić-Stojanović et al. 2009; Salonen et al. 2010). The phylogenetic HITChip microarray

118 targets the V1 and V6 hypervariable regions of the 16S rRNA gene of over 1000 bacterial phylotypes

119 that present the majority of the so far detected phylotypes of the human intestine. Phylogenetic 120 organization of the microarray probes and data preprocessing have been explained in detail elsewhere

121 (Rajilić-Stojanović et al. 2009; Salonen et al. 2010; Jalanka-Tuovinen et al. 2011). Quantification of

U122

123

124

125

126

127

128

129

130

131

132

133

134

135

136

137

138

139

the relative differences in the taxon abundance between samples was obtained by summarizing the

probe signals to phylotype (species-like), genus and phylum levels. Genus-level taxa with $\geq 90 \%$

sequence similarity in the $16 \mathrm{~S}$ rRNA gene are referred to as Species and relatives, the latter being shortened in the text as “et rel.” (Rajilić-Stojanović et al. 2009).

Previously, HITChip-derived microbial profiles have been shown to correlate well with those obtained with fluorescence in situ hybridization (FISH) (Rajilić-Stojanović et al. 2009) and pyrosequencing (Claesson et al. 2009). Samples were also used for absolute quantification of total bacteria, methanogenic Archaea, Lactobacillus group and Bifidobacterium spp. with previously described qPCR primers and reaction conditions (Salonen et al. 2010).

\section{Blood samples and their biochemical analyses}

Of the 25 study subjects used for the microbiota analysis, 22 (8 from probiotic group and 14 from placebo group) were available for the parallel analysis of serum lipid profiles before and after the intervention (44 samples in total). Venous blood samples from the antecubital vein were taken at baseline and after the three-week intervention. The blood samples were stored at $-20^{\circ} \mathrm{C}$ for further analyses. Total cholesterol, high-density lipoprotein (HDL) and low-density lipoprotein (LDL) cholesterol as well as triglyceride levels were enzymatically determined from the serum as previously described (Kekkonen et al. 2008a). 
141 Global lipid profiling: sample preparation and analysis by UPLC-MS

142 Extraction of lipids from the serum samples, analysis of the lipid extracts with Waters Q-Tof Premier

143 mass spectrometer combined with an Acquity Ultra Performance LC ${ }^{\mathrm{TM}}$ (UPLC), data preprocessing and

144 identification of the lipid molecular species is described in detail elsewhere (Kekkonen et al. 2008a).

145 Lipids have been named according to Lipid Maps (http://www.lipidmaps.org) with the following

146 abbreviations: Cer: ceramide; ChoE: cholesteryl ester; lysoPC: lysophosphatidylcholine; PA:

147 phosphatidic acid; PG: phosphatidylglycerol; PC: phosphatidylcholine; PS: phosphatidylserine; SM:

148 sphingomyelin; TG: triglyceride. Where the fatty acid composition could not be determined, the total

149 number of carbons and double bonds is indicated. The first number indicates the amount of carbon

150 atoms in the fatty acid molecule, followed by the number of double bonds. For details, see (Kekkonen

151 et al. 2008a).

152

153 Statistical analyses

154 The effects of probiotic intervention on the intestinal microbiota were investigated with a combination

155 of linear models, sparse principal component analysis (PCA; Shen \& Huang 2008; Lê Cao, González \&

156 Dèjean 2009), unsupervised hierarchical clustering, and significance testing. The log-transformed

157 HITChip and lipid profiling values were approximately Gaussian distributed and fulfilled the general

158 statistical assumptions underlying the selected computational approaches. Background variables,

159 including age, body-mass index and gender were compared in the baseline samples to exclude 160 potentially confounding effects associated with these variables. The Wilcoxon test was used with 161 continuous variables and the Fisher exact test for categorical variables, followed by

162 Benjamini-Hochberg p-value correction for multiple testing. No significant differences between the 163 background variables were observed between the treatment groups ( $p>0.05$ in all comparisons). The 
164 two-group comparisons between the time points and between the treatment groups were quantified 165 based on a linear model with group-wise fixed effects and sample-specific random effects, as 166 implemented in the limma R package (Smyth 2004), to identify bacterial taxa with significant changes 167 induced by the probiotic intervention and to assess the magnitude and significance of the effects. The 168 function lmFit was used to fit the linear model, followed by significance estimation by empirical Bayes 169 as described in Smyth (2004). In addition, power calculation was carried out to assess statistical power U170 of the current study. The original data was randomly permuted, one significant 2-fold alteration was 171 inserted, and Gaussian noise was added using the same average standard deviation as in the original 172 data. Empirical power calculation with 1000 random permutations showed that 2-fold and higher 173 alterations that follow the noise levels in the original data were detected in $>99.8 \%$ of the cases with 174 sample size of 8 or more, based on the same detection criteria than in the current study, confirming the 175 high statistical power of the analysis at the present sample size. PCA is a linear dimension reduction 176 method used to compress information in the high-dimensional phylogenetic (genus-level) and lipid 177 profiles into few informative features that capture the main variation in the data and allow 178 two-dimensional visualization of sample similarities. The temporal and inter-individual similarity of 179 the microbiota and lipid profiles was assessed by average intra- and inter-individual Pearson 180 correlations (r) between and within the time points, respectively. The differences in profile similarity

181 between the probiotic and placebo groups were estimated with Wilcoxon test. The biweight 182 midcorrelation, which is more robust to outliers than the standard Pearson correlation, was used to 183 quantify correlation between the microbiota and lipid profiles across the individuals. High-throughput 184 screening studies involve considerable multiple testing and the traditional multiple testing correction 185 approaches are prohibitively conservative in this context due to their emphasis on estimating the 186 probability of a single false positive finding. Hence, we have used q-values for multiple testing 
187 correction in the high-throughput screening tests that include parallel comparisons of large numbers of

188 lipids and bacterial phylotypes.

189 Correlation analysis of the intestinal microbiota and serum lipids

190 The biweight midcorrelation measure was used to quantify associations between the microbiota and 191 lipid profiling data sets, and between the microbiota and biochemically determined lipids. The 192 correlations between genus-level bacterial groups and lipid species were calculated together with 193 significance estimates that were corrected for multiple testing. The significantly correlated $(\mathrm{q}<0.05)$

194 phylotypes and lipids were selected for further analysis. It is important to note, however, that while the 195 present sample size of 22 samples across 2 time points (before and during the probiotic intervention) is 196 sufficient for highlighting significant correlation patterns, the present experimental design cannot 197 uncover causal relationships and the observed correlations may be partly associated with diet or other 198 confounding variables that simultaneously affect both lipid and microbiota profiles. Two-way average 199 linkage hierarchical clustering of the bacterial taxa and lipids was applied to highlight and visualize 200 groups of phylotypes and lipids sharing similar correlation patterns. Analogous 'second-order' 201 correlations have previously been applied for instance in the context of gene expression studies 202 (Parmigiani et al. 2004; Lahti et al. 2012). A constant plaid model biclustering was used for systematic 203 detection of significantly correlated bacterial and lipid groups on the correlation heatmap (Lazzeroni \& 204 Owen 2002). To interpret the detected biclusters, statistical enrichment (over-representation) of the 205 implicated bacteria was quantified. Moreover, enrichment analysis was carried out for lipids containing 206 even or odd number of carbon atoms, as well as the enrichment of lipids with zero, one or more double 207 bonds. The enrichment analyses were carried out with Fisher's exact test (Rivals et al. 2007). All 208 analyses were performed within the R statistical environment (R Development Core Team 2010). 


\section{Results}

211 Impact of the L. rhamnosus GG intervention in the intestinal microbiota

212 This study characterized the impact of L. rhamnosus GG intervention on the stability and composition

213 of the intestinal microbiota. The subjects in the probiotic group consumed daily approximately $10^{10}$

214 (10.2 $\left.\log _{10}\right)$ colony forming units (cfu) of L. rhamnosus GG. The compliance was verified with the

215 quantification of L. rhamnosus GG in the feces with strain-specific qPCR (Kekkonen et al. 2008b). The

216 average excretion of $L$. rhamnosus GG in the probiotic group was more than 1000 -fold higher than that

217 in the placebo group (8.52; sd $0.73 \log _{10}$ versus 5.20 ; sd $1.09 \log _{10}$ genome copies per gram of feces, 218 respectively).

The stability of the microbiota during the trial was quantified by the similarity of the

220 microbiota profiles between the three time points with Pearson correlation (Table 1). The average 221 intra-individual correlations were high; 0.94-0.95 (sd 0.02-0.03). No significant difference in the 222 temporal stability of the microbiota between the probiotic and placebo groups was observed, indicating 223 that the probiotic intervention did not alter the overall microbial stability. Principal Component 224 Analysis (PCA) visualization of the relationships between the intestinal microbiota profiles 225 (Supplementary Fig. S1A) and hierarchical clustering (data not shown) further supported the 226 conclusion that there were no systematic differences in the microbiota between the intervention groups.

227 The average inter-individual microbiota correlation was 0.76-0.78 with no significant differences 228 between the probiotic and placebo groups (Table 1). The intra-individual microbiota correlations 229 ( $\mathrm{r}=0.94-0.95)$ were notably higher than the inter-individual correlations ( $\mathrm{r}=0.76-0.78)$, stressing the 230 subject-specificity of the microbiota.

231 Linear models were used to quantify the effects of the L. rhamnosus GG intervention on 232 individual taxa using genus- and phylotype-level microarray data. A specific and transient increase of 
233 bacteria related to L. rhamnosus was detected in the probiotic group immediately after the intervention

234 (Fig. 1). There were no intervention-related effects in the other lactobacilli, Bifidobacteria or any other 235 taxa either in the probiotic or placebo group; however substantial individual variation was evident.

236 To complement the HITChip microarray analysis, we also determined the absolute counts of 237 total bacteria, methanogenic Archaea, Lactobacillus group and Bifidobacterium spp. using real-time 238 PCR. The ingestion of $L$. rhamnosus $G G$ was reflected in the total lactobacilli that showed a highly significant increase in the probiotic group after the intervention, returning to baseline levels in the follow up ( $q<0.05$ in both comparisons). No other significant changes were observed in the amount of targeted microbes neither in the probiotic or placebo group. We detected substantial inter- and intra-individual variation in the methanogenic Archaea but that was independent of the time point or

243 the treatment group (data not shown). These observations support the conclusion that the L. rhamnosus

244 GG intervention did not change the overall microbiota composition.

\section{High-throughput profiling of serum lipids}

247 Global profiling of the serum lipids was performed using the UPLC-MS platform to assess the serum 248 lipid profiles at the molecular species level (Kekkonen et al. 2008a). In total, 407 lipid species from 11 249 different classes were identified. The lipid profiles were dominated with triglycerides (TG; 37\%), 250 phosphatidylcholines (PC; 25\%) and phosphatidyletanolamines (PE; 13\%) while phosphatidic acids 251 (PA), phosphatidylglycerols (PG), sphingomyelins (SM), cholesteryl esters (ChoE), 252 lysophosphatidylcholines (lysoPC), phosphatidylserines (PS), ceramides (Cer) and 253 lysophosphatidylethanolamines (lysoPE) each contributed with $7 \%$ to $0.3 \%$ to the total lipid pool. 
256 profile stability were observed between the treatment groups (average intra-individual $\mathrm{r}=0.92$ and $257 \mathrm{r}=0.93$ for the probiotic and placebo groups, respectively; Table 1). In contrast to microbiota profiles,

258 the serum lipid profiles were remarkably similar, not only within the subjects but also between the 259 subjects (average inter-individual $\mathrm{r}=0.89-0.91$; Table 1 ). No significant differences were seen between 260 the probiotic and placebo groups, or between the time points. The stability of the lipid and microbiota 261 profiles showed a weak positive correlation $(\mathrm{r}=0.27)$ across the subjects independent of a treatment group. The global lipid profiles did not separate according to the treatment group in PCA visualization (Supplementary Fig. S1B), and no statistically significant lipid alterations were detected between the time points neither in the probiotic or placebo group (data not shown; Kekkonen et al. 2008b). The fold-changes for each lipid species between the two time points followed normal distribution in both

266 intervention groups (data not shown), which further supports the conclusion that the intervention did 267 not induce systematic alterations on the lipid profiles.

Associations between the intestinal microbiota and serum lipids

In addition to studying the effects of probiotic L. rhamnosus $G G$ intervention in the intestinal 271 microbiota, we investigated the co-variation of the microbiota and serum lipid profiles. As discussed 272 above, our results (Table 1, Supplementary Fig. S1) conclusively show that the probiotic intervention 273 did not affect the lipid profiles or the microbiota beyond the L. rhamnosus that was ingested. Hence, 274 the subjects from probiotic and placebo groups were pooled to increase the sample size and statistical 275 power in exploring the associations between the intestinal microbiota and serum lipids. Heatmap visualization of the microbiota-lipid correlations across the 22 subjects from 2 time 277 points revealed intestinal bacteria and serum lipids with significantly correlated abundance patterns 278 (Fig. 2). In total, 86 bacterial group-lipid pairs with notable correlations (+/- 0.5 or higher) were 
279 identified at $\mathrm{q}<0.05$ significance level (Fig. 2). Among the significant correlations, 23 of the 131 280 genus-level taxa detectable by the HITChip were represented (Supplementary Table S3). Most of these 281 taxa belonged to Proteobacteria (6) and Firmicutes within Clostridium cluster XIVa (5) and Bacilli (3). 282 From the lipid side, the vast majority of the significant correlations were attributable to the most 283 dominant lipids, TGs (62\%) and PCs (30\%). When analyzed at the genus-level, the significant 284 correlations were strongly dominated by the positive correlations between bacteria related to

Ruminococcus gnavus and different TG species (31 of 40 significant positive correlations, average $\mathrm{r}=0.61$; Fig. 3A). Detailed analysis at the phylotype-level data indicated that within the $R$. gnavus group, two uncultured phylotypes, uncultured human gut bacterium JW1G3 and JW1H4a, dominated the correlations while the type species $R$. gnavus or related $R$. torques did not correlate significantly with TG or any other lipid species. Other representatives of the Clostridium cluster XIVa included bacteria related to Dorea formicigenerans that also correlated positively with four TG species (Fig. 2; average $\mathrm{r}=0.58$ ). The remaining TG correlations were negative and involved phylogenetically diverse bacteria (Supplementary Table S3). Also PCs correlated significantly with numerous taxa, most of which correlated negatively with ester- or ether linked PCs. Interestingly, a set of Proteobacteria (Campylobacter, Helicobacter and Moraxellaceae) with low signal intensities had a clear negative correlation with PC. Similarly, two Lactobacillus species (L. rhamnosus that was ingested during the trial and L. salivarius) together with another group of Bacillus, Enterococcus spp., also correlated negatively with PC while few Firmicutes exhibited a positive correlation with this lipid species. Among the less abundant lipids ( $<10 \%$ of the total lipid pool), a positive correlation was detected between cholesteryl ester and Collinsella (r=0.59; Supplementary Table S3, Fig. 4A). On the phylotype level, the correlation was mainly attributable to an uncultured bacterium clone Eldhufec074 within genus Collinsella. Another group of Actinobacteria, namely Actinomycetaceae, showed a significant negative correlation to TG and PA. 
304 pairs revealed groups of lipids and bacteria sharing similar correlation patterns. A constant plaid model

305 biclustering approach was applied to detect coherent groups of significantly correlating microbe-lipid

306 pairs (see Methods). The analysis revealed three major clusters of significantly correlated lipid-microbe

307 pairs (Supplementary Table S2). One of the observed biclusters highlights the above discussed positive

308 association between bacteria related to $R$. gnavus and TGs. Another TG cluster, containing highly unsaturated long-chain fatty acids, consisted of negatively correlating bacteria related to Megamonas hypermegale (family Veillonellaceae, Clostridium cluster IX) or belonging to the Actinomycetaceae.

311 The third cluster contained a set of Proteobacteria (Helicobacter, Oceanospirillum and Moraxellaceae 312 spp.) and bacteria related to Eubacterium cylindroides within the family Erysipelotrichaceae, which all 313 correlated negatively with ether lipids.

To explore the nature of the identified lipid-microbe pairs, we quantified the enrichment of specific, functionally relevant lipid categories in the biclusters. In particular, we investigated the enrichment of lipids with even and odd number of carbon atoms and the degree of saturation $(0,1$, or

317 more double bonds). The origin of lipid can be inferred from its chemical composition: Polyunsaturated 318 fatty acids (PUFAs; more than one double bond) and lipids that have uneven number of carbon atoms 319 originating from plants, bacteria or marine organism as humans cannot synthesize them. Significant 320 enrichment of long acyl chain polyunsaturated fatty acids (two or more hydrogen bonds; $<<0.05$; Fisher 321 exact test) was detected among the TG lipids that significantly correlated with bacteria related to $R$. 322 gnavus (Fig. S2; Supplementary Table S2). Such a positive association suggests a role for these $R$. 323 gnavus-related bacteria in the absorption of dietary lipids. In support of this interpretation, TGs 324 containing fatty acids with odd number of carbons were also relatively common in this group ( $\mathrm{p}=0.1)$. 325 In the other two biclusters, no significant enrichment of odd/even carbon count or saturation level was 
326 detected but within the bicluster 2 (Supplementary Table S2), Actinomycetaceae correlated exclusively 327 with highly unsaturated PUFAs (Fig. 2).

329 Associations between the intestinal microbiota and biochemically determined serum lipids

330 The mean values (SD) for the major serum lipids were total cholesterol 5.10 (1.02), LDL cholesterol 3313.00 (1.21), HDL cholesterol 1.50 (0.33) and TG 1.20 (0.71) mmol/L (Kekkonen et al. 2008a). No 332 significant changes were detected in the lipids of the probiotic or the placebo groups during the 333 intervention (Kekkonen et al. 2008b). Hence, we analyzed the potential associations of the 334 enzymatically determined major blood lipids with the intestinal microbiota (Table 2). A positive 335 correlation between bacteria related to $R$. gnavus and TG was observed ( $(\mathrm{q}<0.01 ; \mathrm{r}=0.60$; Fig. 3B), 336 corroborating the association identified within the global lipid analysis. In line with the spectroscopic 337 lipid analysis, representatives of Bacteroidetes and Uncultured Clostridiales correlated negatively and 338 other implicated Firmicutes positively with enzymatically determined TG (Table 2). Representatives of 339 Proteobacteria and Actinobacteria that correlated negatively with TG in the lipid profiling data did not 340

341 methodological reasons as the enzymatic assay captures not only TG but also diacylglyceride, 342 monoacylglyceride and free glycerol, while lipid profiling captures TGs at the molecular level. Collinsella spp. and Eubacterium biforme et rel. showed statistically significant $(\mathrm{q}<0.05)$ and 344 positive correlations to enzymatically determined total and LDL cholesterol (Fig. 4B; Supplementary 345 Table S2). No other significant correlations were identified for total or LDL cholesterol while HDL 346 cholesterol correlated significantly with numerous taxa. Eight different Firmicutes including taxa 347 related to Ruminococcus obeum and D. formicigenerans were found to correlate negatively with HDL, 
348 while Uncultured Clostridiales I was the only taxon showing positive correlation to HDL

349 (Supplementary Table S2).

351 Discussion

352 In the present study, we analyzed the effect of probiotic intake on the stability and composition of the 353 intestinal microbiota and of the serum lipids, and the overall associations between the microbiota and

354 lipid profiles. The microbiota was analyzed using the HITChip, a phylogenetic microarray, providing 355 one of the first holistic and community-level microbiota assessments after a probiotic intervention. The 356 data published so far is largely dominated with targeted microbiota analyses that have reported a 357 generic increase of lactic acid bacteria after intake of individual lactobacilli strains (McNulty et al. 358 2011; Palaria, Johnson-Kanda \& O'Sullivan 2011; Satokari et al. 2001; Savard et al. 2011; Vaughan, 359 Mollet \& de Vos 1999; Yamano et al. 2006). Our community-level analysis showed that the probiotic 360 intake did not introduce any changes in the microbiota composition or stability except the specific 361 increase of L. rhamnosus and total lactobacilli, which likely reflected the excretion of the ingested 362 strain. Similarly, ingestion of a mixture of five different probiotic strains did not induce any significant 363 changes in the microbiota composition neither in adults nor in simplified model community in mice 364 (McNulty et al. 2011). Even when a combination of probiotic and prebiotic (symbiotic) food was 365 consumed, two studies based on microbiota profiling and qPCR did not identify any differences in the 366 microbiota composition between placebo and treatment besides the ingested strains (Palaria, 367 Johnson-Kanda \& O'Sullivan 2011; Vitali et al. 2010).

We have shown previously using the same cohort that the ingestion of L. rhamnosus GG had 369 apparent effects on the host immunology (Kekkonen et al. 2008b). Hence, our results does not support 370 the hypothesis that probiotic bacteria would modulate the endogenous microbiota but rather points 
371 towards direct signaling to host or altered gene expression of the resident microbiota as a mode of 372 probiotic action as recently proposed (McNulty et al. 2011).

Our study was carried out in healthy adults with seemingly well-established and balanced

374 microbial communities. In subjects whose intestinal ecosystem is unbalanced e.g. due to pathogen

375

376 overgrowth, gastrointestinal symptoms or recent intake of antibiotics, intake of probiotic bacteria may modulate the microbiota. Similarly, the developing microbiota of children is potentially more susceptible to environmental modulators including probiotics. Although substantial changes in infant microbiota following daily intake of L. rhamnosus GG have indeed been reported (Cox et al. 2010), these results must be interpreted with caution as the used phylogenetic microarray provided signals for close to 50 phyla whereas the human intestinal ecosystem only contains a maximum of 10 phyla (Rajilić-Stojanović, Smidt \& de Vos 2007; Midgley et al. 2012).

To the best of our knowledge, this cohort is the first addressing the potential impact of probiotics on global serum lipid profiles in adults. Daily intake of $L$. rhamnosus $G G$ for three weeks did not introduce any consistent alterations in the serum lipids or in the biochemically determined cholesterol and triglyceride levels of the healthy, normolipidemic adults analyzed in this and previous study (Kekkonen et al. 2008b). Probiotic bacteria have been suggested as a potential non-drug treatment to lower serum cholesterol levels based on their in vitro described ability to deconjugate bile acids and directly assimilate cholesterol. A recent meta-analysis implies that probiotic intake can lower the total and LDL cholesterol (Guo et al. 2011), although numerous human studies have failed to observe any effects on serum lipids after probiotic intake (see e.g. Sadrzadeh-Yeganeh et al. 2010; Pereira \& Gibson 2002). One explanation for the controversy is that similarly to the strain-specificity of the immunomodulatory effects of probiotics (Kekkonen et al. 2008b), also their effects on lipid metabolism are likely to vary between different strains. In atopic infants the consumption of $L$. 
394 rhamnosus GG supplemented formulas for several months resulted in a reduced proportion of $395 \alpha$-linolenic acid and of total n-3 PUFAs, leading to increased n-6 to n-3 PUFA ratio in sera of 396 probiotic-fed infants (Kankaanpää et al. 2002). Decrease of n-3 fatty acids has negative health 397 implication, underlining the need for more in vivo research to specify the bacterial strains and target 398 population where probiotics can have beneficial effects on the host lipid metabolism.

Our data indicates that the variation of the intestinal microbiota is considerably higher across the individuals than the variation in serum lipid profiles, highlighting the tight homeostatic control of systemically circulating lipids. The intestinal microbiota is known to play an important role in the regulation of systemic lipid metabolism (Martin et al. 2007; Velagapudi et al. 2010). In this study, we identified several novel associations between the human intestinal commensals and serum lipids. From the lipid side, TGs (62\%) and PCs (30\%) dominated the significant lipid-microbe correlations. TGs are used for energy storage while PCs are abundant constituents of cell membranes. Both of these abundant lipid classes were affected upon colonization of germ-free mice (Velagapudi et al. 2010). The most prominent correlation was between TGs and uncultured phylotypes related to R. gnavus that belong to 408 family Ruminococcaceae in Clostridium cluster XIVa. Two characterized species within the group, $R$. 409 gnavus and $R$. torques, have been implicated in intestinal disorders as they appeared to be 410 overrepresented both in IBS (Kassinen et al. 2007; Rajilić-Stojanović et al. 2011) and in IBD (Joossens

411 et al. 2011; Prindiville, Cantrell \& Wilson. 2004). The TG lipids that were positively associated with $R$. 412 gnavus-related phylotypes were enriched with polyunsaturated and odd-chain fatty acids. Since the 413 odd-chain fatty acids are not synthesized in the body, these positive associations suggest that the 414 implicated bacteria facilitate the absorption of polyunsaturated dietary lipids. Moreover, it cannot be 415 excluded that these bacteria are even involved in their biosynthesis as related $R$. obeum together with 416 other Firmicutes are among the intestinal bacteria capable of producing isomers of conjugated linoleic 417 acids (CLA; McIntosh et al. 2009). 

same pattern regarding microbial correlations. For example, bacteria related to D. formicigenerans showed positive association to saturated triglycerides with a small carbon number, i.e. including

421 palmitic acid. These fatty acids are mainly produced de novo in the liver (Westerbacka et al. 2010;

422 Kotronen et al. 2009). D. formicigenerans produces formate and acetate that are precursors of hepatic

423 lipogenesis. Recent metaproteomic work verifies that the acetate kinases involved in acetate production are highly expressed in the intestinal ecosystem (Kolmeder et al. 2012), suggesting that several intestinal bacteria have potential to regulate lipogenesis. On the other hand, Actinomycetaceae were inversely associated with TGs containing highly unsaturated triglycerides with a high carbon number.

427 Such TGs carry physiologically important fatty acids such as C22:6 (docosahexanoic acid) and C20:4 428 (arachidonic acid). Both are important cell membrane components, the former especially in visual and 429 neural tissues, and the latter is involved in inflammatory signaling. While not much is known about the 430 intestinal Actinomycetaceae, both commensal and pathogenic forms of these Actinobacteria are 431 abundant in the oral cavity. We identified multiple taxa correlating negatively with normal or ether linked forms of PC. 433 Dietary PC was recently identified as compound that after conversion by the intestinal microbiota 434 promotes heart disease (Wang et al. 2011). Unfortunately, that study did not address the identity of 435 bacteria involved in the metabolism of dietary PC, but our data suggest that different Firmicutes, 436 Proteobacteria and Fusobacteria may participate in this metabolic conversion (Fig. 2). Remarkably, the 437 presence of serum ether PCs was negatively associated with various taxa, mainly with Gram-negative 438 genera that include pathogens and commensals, such as Helicobacter, Moraxellaceae and 439 Campylobacter. Among the ether lipids, plasmalogens, known as endogenous antioxidants (Wallner \& 440 Schmitz 2011), are the most abundant. Their negative association with Proteobacteria could indicate 441 that the implicated bacteria may induce oxidative stress in host cells, leading to depletion of 
442 plasmalogens. In support of this hypothesis, lipopolysaccharide (LPS), a ubiquitous and toxic surface 443 component of Gram-negative bacteria, induces oxidative stress in mammalian cell cultures (Aly, 444 Lightfoot \& El-Shemy 2010).

445 Finally, we identified positive correlation between the abundance of serum cholesterol and 446 genus Collinsella (Actinobacteria, family Coriobacteriacea). Biochemical lipid analysis indicated that 447 Collinsella spp. together with bacteria related to E. biforme correlated specifically with total cholesterol

448

449

450

451

452

453

454

455

456

457

458

459

460

461

462

463

464

465

and LDL but not HDL. To our knowledge, our work provides the first in vivo evidence about the implication of Coriobacteriacea in human lipid metabolism. Our present findings are supported by the data generated with rodent models. In a hamster model, the proportion of Coriobacteriaceae showed high positive correlation with non-HDL plasma cholesterol levels and reacted to the intake of dietary lipids (Martinez et al. 2009). In a mouse model, a strong positive correlation between Coriobacteriaceae and hepatic triglycerides was identified (Claus et al. 2011), providing cumulative indication for the involvement of Coriobacteriaceae in mammalian lipid metabolism. While only one taxon (Collinsella) correlated statistically significantly with cholesterol in the lipid profiling data, numerous taxa showed significant correlation to enzymatically determined HDL cholesterol. This may arise from technical differences, as the lipid profiling does not quantify free cholesterol but its derivative cholesteryl ester, where cholesterol is esterified to long-chain fatty acids. All taxa that were associated with both HDL cholesterol and TG had opposite correlation with these lipids (Supplementary Table S2), in accordance with the fact that serum TG and HDL show strong inverse relationship, and their ratio is used as an atherogenic index that predicts cardiovascular risk (Jeppesen et al. 1997). Hence, identification of bacteria that potentially affect the athreogenic index may provide important clinical implications for dyslipidemic individuals.

While good correspondence between the HITChip and pyrosequencing studies have been previously demonstrated, the main advantage of the HITChip microarray compared to the 
466

467

468

469

470

471

481 482

483

484 485 486 487 488 489

pyrosequencing studies is that the microarray technology provides very standardized and cost-efficient tools for deep and reproducible analysis of intestinal microbiota including phylotypes that are only present in low concentrations (Claesson et al., 2009; Salonen et al. 2012). We expect that our major findings, the associations between specific lipid species and bacteria related to Collinsella or $R$. gnavus could be detected also in a standard pyrosequencing study due to the relatively high abundance of these organisms. However, the associations involving less abundant taxa (Bacilli, Proteobacteria, Actinomycetaeae) would likely have been missed with conventional sequencing depth.

In summary, quantitative analysis of high-throughput profiling data identified several significant correlations between the intestinal microbiota and serum lipids. These results partly confirm and extend previous observations in animal studies, and provide hypotheses for follow-up studies in humans. It is important to note, however, that the present analysis is based on a Finnish cohort and only two time points, and does not consider causal relationships between these variables. Therefore it cannot be excluded that some of the observed correlations may be associated with the diet or other confounding variables that simultaneously affect both lipid and microbiota profiles. However, as all subjects ate their habitual diets, individual food components are not likely to cause systematic bias to the observed lipid-microbe correlations, which is further supported by the observation of distinct groups of correlated lipid-microbe pairs in the bicluster analysis. It can also be ruled out that the intervention would have caused the correlations as control measurements from the baseline were included, the ingested probiotics L. rhamnosus GG did not correlate significantly with any serum lipid, and the drink did not otherwise alter the microbiota. Confirmation of the findings in an independent cohort and a more thorough longitudinal analysis will be necessary to assess the effect of external variables on the microbiota-lipid correlations and their potential causal associations. None of the implicated bacterial taxa are functionally characterized, and thus the potential mechanisms of how human intestinal bacteria relate to the host lipid metabolism are currently unknown. Among the 
490 potential mechanisms, bacterial modification of the bile acid pool is by far the best characterized.

491 Collinsella spp., $R$. gnavus as well as the genus Eubacterium are among the intestinal bacteria capable 492 of deconjugating bile acids (Lepercq et al. 2004). Bile acids are cholesterol-derived detergents that play

493 a central role in the absorption of fat in the intestine but also in signaling with systemic endocrine 494 functions (Swann et al. 2011). It has been known for long that intestinal bacteria can chemically 495 modify bile acids but recent work suggest that the microbiota may also control their production and 496 degradation (Antunes et al. 2011). In mice the intestinal bacteria profoundly affect the emulsification, 497 absorption and transport of dietary fat as well as their storage and peroxidation through the metabolic 498 and signaling properties of bile acids (Martin et al. 2007).

\section{Conclusion}

501 Our data supports the concept that the overall lipid content in human serum is a composite of host and microbial metabolic activity, and the intestinal commensals are implicated in the metabolism of various 503 lipid species that human body utilizes for membranes, energy storage and signaling. Considering that a 504 single gene in an intestinal bacterium could alter host fatty acid composition (Rosberg-Cody et al. 505 2011), we can only envisage the metabolic capacity and the functional consequences from the million 506 genes in the intestinal microbiome. As epidemiological data do not support a link between dietary 507 cholesterol and serum cholesterol levels (Lecerf \& de Lorgeril 2011), the role of genetic factors in the 508 individual variability of cholesterol levels is evident. Identification of the bacteria and their 509 mechanisms that regulate host fatty acid and lipid metabolism have considerable potential for clinical 510 implications due to the profound role of these molecules for instance in cardiovascular disease and 511 regulation of inflammatory cytokine signaling (Calder 2011). Future trials involving controlled diet and 
512 dyslipidemic individuals will provide further insights on the role of intestinal microbes on human lipid 513 metabolism.

514

515 References

516 Ahlroos T, Tynkkynen S. 2009. Quantitative strain-specific detection of Lactobacillus rhamnosus GG

517 in human faecal samples by real-time PCR. Journal of Applied Microbiology 106:506-514.

518 Aly HA, Lightfoot DA, El-Shemy HA. 2010. Bacterial lipopolysaccharide-induced oxidative stress in

519 adult rat Sertoli cells in vitro. Toxicology in Vitro 24:1266-1272.

520 Antunes LCM, Han J, Ferreira RBR, Lolić P, Borchers CH, Finlay BB. 2011. Effect of antibiotic 521 treatment on the intestinal metabolome. Antimicrobial Agents and Chemotheraphy 55:1494-1503.

522 Blaut M, Clavel T. 2007. Metabolic diversity of the intestinal microbiota: implications for health and 523 disease. The Journal of Nutrition 137:751S-755S.

524 Calder P. 2011. Fatty acids and inflammation: the cutting edge between food and pharma. European 525 Journal of Pharmacology 668:S50-58.

526 Claesson MJ, O'Sullivan O, Wang Q, Nikkilä J, Marchesi JR, Smidt H, de Vos VM, Ross RP, O'Toole 527 PW. 2009. Comparative analysis of pyrosequencing and a phylogenetic microarray for exploring 528 microbial community structures in the human distal intestine. PLoS One 4:e6669.

529 Claus SP, Ellero SL, Berger B, Krause L , Bruttin A, Molina J, Paris A, Want EJ, de Waziers I, Cloarec 530 O, Richards SE, Wang Y, Dumas M-E, Ross A, Rezzi S, Kochhar S, van Bladeren P, Lindon JC, 531 Holmes E, Nicholson JK. 2011. Colonization-Induced Host-Gut Microbial Metabolic Interaction. mBio 532 2:e00271-00210. 
533 Cox MJ, Huang YJ, Fujimura KE, Liu JT, McKean M, Homer Boushey HA, Segal MR, Brodie EL,

534 Cabana MD, Lynch SV. 2010. Lactobacillus casei abundance is associated with profound shifts in the 535 infant gut microbiome. PLoS One 5:e8745.

536 Fava F. 2006. The gut microbiota and lipid metabolism: implications for human health and coronary 537 heart disease. Current Medicinal Chemistry 13:3005-3021.

538 Gérard P. 2010. Gastrointestinal Tract: Microbial Metabolism of Steroids. In: Timmis KN, ed.: 539 Handbook of Hydrocarbon and Lipid Microbiology. Springer Berlin Heidelberg. 3133-3140.

540 Guo Z, Liu XM, Zhang QX, Shen Z, Tian FW, Sun ZH, Zhang HP, Chen W. 2011. Influence of 541 consumption of probiotics on the plasma lipid profile: a meta-analysis of randomised controlled trials. 542 Nutrition, Metabolism and Cardiovascular Diseases 21:844-850.

543 Holmes E, Li JV, Athanasiou T, Ashrafian H, Nicholson JK. 2011. Understanding the role of gut 544 microbiome-host metabolic signal disruption in health and disease. Trends in Microbiology 19:349-359.

545 Jalanka-Tuovinen J, Salonen A, Nikkilä J, Immonen O, Kekkonen R, Lahti L, Palva A, de Vos WM. 546 2011. Intestinal microbiota in healthy adults: temporal analysis reveals individual and common core 547 and relation to intestinal symptoms. PLoS One 6:e23035.

548 Jeppesen J, Hein HO, Suadicani P, Gyntelberg F. 1997. Relation of high TG-low HDL cholesterol and 549 LDL cholesterol to the incidence of ischemic heart disease. An 8-year follow-up in the Copenhagen 550 Male Study. Arteriosclerosis, Thrombosis and Vascular Biology 17:1114-1120.

551 Joossens M, Huys G, Cnockaert M, de Preter V, Verbeke K, Rutgeerts P, Vandamme P, Vermeire S. 552 2011. Dysbiosis of the faecal microbiota in patients with Crohn's disease and their unaffected relatives. 553 Gut 60:631-637. 
554 Kankaanpää PE, Yang B, Kallio HP, Isolauri E, Salminen SJ. 2002. Influence of probiotic 555 supplemented infant formula on composition of plasma lipids in atopic infants. The Journal of 556 Nutritional Biochemistry 13:364-369.

557 Kankainen M, Paulin L, Tynkkynen S, von Ossowski I, Reunanen J, Partanen P, Satokari R, Vesterlund 558 S, Hendrickx APA, Lebeer S, De Keersmaecker SCJ, Vanderleyden J, Hämäläinen T, Laukkanen S, 559 Salovuori N, Ritari J, Alatalo E, Korpela R, Mattila-Sandholm T, Lassig A, Hatakka K, Kinnunen KT, 560 Karjalainen H, Saxelin M, Laakso K, Surakka A, Palva A, Salusjärvi T, Auvinen P and de Vos WM. 561 2009. Comparative genomic analysis of Lactobacillus rhamnosus GG reveals pili containing a 562 human-mucus binding protein. Proceedings of the National Academy of Sciences $U S A$ 563 106:17193-17198.

564 Kassinen A, Krogius-Kurikka L, Mäkivuokko H, Rinttilä T, Paulin L, Corander J, Malinen E, 565 Apajalahti J, Palva A. 2007. The fecal microbiota of irritable bowel syndrome patients differs 566 significantly from that of healthy subjects. Gastroenterology 133:24-33.

567 Kekkonen RA, Sysi-Aho M, Seppänen-Laakso T, Julkunen I, Vapaatalo H, Orešič M, Korpela R. 568 2008a. Effect of probiotic Lactobacillus rhamnosus GG intervention on global serum lipidomic profiles 569 in healthy adults. World Journal of Gastroenterology 14:3188-3194.

570 Kekkonen RA, Lummela N, Karjalainen H, Latvala S, Tynkkynen S, Järvenpää S, Kautiainen H, 571 Julkunen I, Vapaatalo H, Korpela R. 2008b. Probiotic intervention has strain-specific anti-inflammatory 572 effects in healthy adults. World Journal of Gastroenterology 14:2029-2036.

573 Kolmeder CA, de Been M, Nikkilä J, Ritamo I, Mättö J, Valmu L, Salojärvi J, Palva A, Salonen A, de 574 Vos WM. 2012. Comparative metaproteomics and diversity analysis of human intestinal microbiota 575 testifies for its temporal stability and expression of core functions. PLoS One 7:e29913. 
576 Kotronen A, Velagapudi VR, Yetukuri L, Westerbacka J, Bergholm R, Ekroos K, Makkonen J, Taskinen

577 MR, Orešič M, Yki-Järvinen H. 2009. Serum saturated fatty acids containing triacylglycerols are better

578 markers of insulin resistance than total serum triacylglycerol concentrations. Diabetologia 52:684-690.

579 Lahti L, Schäfer M, Klein H-U, Bicciato S, Dugas M. 2012. Cancer gene prioritization by integrative 580 analysis of mRNA expression and DNA copy number data: a comparative review. Briefings in

581 Bioinformatics. Online March 2012.

582 Lazzeroni L, Owen A. 2002. Plaid Models for Gene Expression Data. Statistica Sinica 12:61-86.

583 Lê Cao K-A, González I, Dèjean, S. 2009. intergrOmics: an R package to unravel relationships 584 between two omics data sets. Bioinformatics 25:2855-2856.

585 Lecerf JM, de Lorgeril M. 2011. Dietary cholesterol: from physiology to cardiovascular risk. British

586 Journal of Nutrition 106:6-14.

587 Lepercq P, Gérard P, Béguet F, Grill J-P, Relano P, Cayuela C, Juste C. 2004. Isolates from normal

588 human intestinal flora but not lactic acid bacteria exhibit $7 \alpha$ - and $7 \beta$-hydroxysteroid dehydrogenase 589 activities. Microbial Ecology in Health and Disease 16:195-201.

590 Martin FP, Dumas ME, Wang Y, Legido-Quigley C, Yap IK, Tang H, Zirah S, Murphy GM, Cloarec O,

591 Lindon JC, Sprenger N, Fay LB, Kochhar S, van Bladeren P, Holmes E, Nicholson JK. 2007. A 592 top-down systems biology view of microbiome-mammalian metabolic interactions in a mouse model.

593 Molecular Systems Biology 3:112.

594 Martinez I, Wallace G, Zhang C, Legge R, Benson AK, Carr TP, Moriyama EN, Walter J. 2009.

595 Diet-induced metabolic improvements in a hamster model of hypercholesterolemia are strongly linked 596 to alterations of the gut microbiota. Applied Environmental Microbiology 75:4175-4184.

597 McIntosh FM, Shingfield KJ, Devillard E, Russell WR, Wallace RJ. 2009. Mechanism of conjugated

598 linoleic acid and vaccenic acid formation in human faecal suspensions and pure cultures of intestinal 599 bacteria. Microbiology 155:285-294. 
600 McNulty NP, Yatsunenko T, Hsiao A, Faith JJ, Muegge BD, Goodman AL, Henrissat B, Oozeer R, 601 Cools-Portier S, Gobert G, Chervaux C, Knights D, Lozupone CA, Knight R, Duncan AE, Bain JR, 602 Muehlbauer MJ, Newgard CB, Heath AC, Gordon JI. 2011. The Impact of a Consortium of Fermented 603 Milk Strains on the Gut Microbiome of Gnotobiotic Mice and Monozygotic Twins. Science 604 Translational Medicine 3:106.

605 Midgley DJ, Greenfield P, Shaw JM, Oytam Y, Li D, Kerr CA, Hendry P. 2012. Reanalysis and 606 Simulation Suggest a Phylogenetic Microarray Does Not Accurately Profile Microbial Communities. 607 PLoS One 7:e33875.

608 O'Keefe SJ. 2008. Nutrition and colonic health: the critical role of the microbiota. Current Opinion in 609 Gastroenterology 24:51-58.

610 Orešič M, Hänninen VA, Vidal-Puig A. 2008. Lipidomics: a new window to biomedical frontiers. 611 Trends in Biotechnology 26:647-652.

612 Palaria A, Johnson-Kanda I, O'Sullivan DJ. 2011. Effect Of A Synbiotic Yogurt On Levels Of Fecal 613 Bifidobacteria, Clostridia And Enterobacteria. Applied Environmental Microbiology 78:933-940.

614 Parmigiani G, Garrett-Mayer ES, Anbazhagan R, Gabrielson E. 2004. A cross-study comparison of 615 gene expression studies for the molecular classification of lung cancer. Clinical Cancer Research 616 10:2922-2927.

617 Pereira DI, Gibson GR. 2002. Effects of consumption of probiotics and prebiotics on serum lipid levels 618 in humans. Critical Reviews in Biochemistry and Molecular Biology 37:259-281.

619 Prindiville T, Cantrell M, Wilson KH. 2004. Ribosomal DNA sequence analysis of mucosa-associated 620 bacteria in Crohn's disease. Inflammatory Bowel Diseases 10:824-833.

621 Qin J, Li R, Raes J, Arumugam M, Burgdorf KS, Manichanh C, Nielsen T, Pons N, Levenez F, Yamada 622 T, Mende DR, Li J, Xu J, Li S, Li D, Cao J, Wang B, Liang H, Zheng H, Xie Y, Tap J, Lepage P, 
623 Bertalan M, Batto J-M, Hansen T, De Paslier D, Linneberg A, Nielsen HB, Pelletier E, Renault P, 624 Sicheritz-Ponten T, Turner K, Zhu H, Yu C, Li S, Jian M, Zhou Y, Li Y, Zhang X, Li S, Qin N, Yang

625 H, Wang J, Brunak S, Doré J, Guarner F, Kristiansen K, Pedersen O, Parkhill J, Weissenbach J, 626 MetaHIT Consortium, Bork P, Ehrlich SD, Wang J. 2010. A human gut microbial gene catalogue 627 established by metagenomic sequencing. Nature 464:59-65.

628 R Development Core Team. 2010. R: A Language and Environment for Statistical Computing. Vienna, 629 Austria: R Foundation for Statistical Computing.

630 Rajilić-Stojanović M, Smidt H, de Vos WM. 2007. Diversity of the human gastrointestinal tract 631 microbiota revisited. Environmental Microbiology 9:2125-2136.

632 Rajilić-Stojanović M, Heilig HG, Molenaar D, Kajander K, Surakka A, Smidt H, de Vos WM. 2009. 633 Development and application of the human intestinal tract chip, a phylogenetic microarray: analysis of 634 universally conserved phylotypes in the abundant microbiota of young and elderly adults. 635 Environmental Microbiology 11:1736-1751.

636 Rajilić-Stojanović M, Biagi E, Heilig HG, Kajander K, Kekkonen RA, Tims S, de Vos WM. 2011. 637 Global and deep molecular analysis of microbiota signatures in fecal samples from patients with 638 irritable bowel syndrome. Gastroenterology 141:1792-1801.

639 Ridlon JM, Kang DJ, Hylemon PB. 2006. Bile salt biotransformations by human intestinal bacteria. 640 Journal of Lipid Research 47:241-259.

641 Rivals I, Personnaz L, Taing L, Potier M-C. 2007. Enrichment or depletion of a GO category within a 642 class of genes: which test? Bioinformatics 23:401.

643 Rosberg-Cody E, Stanton C, O'Mahony L, Wall R, Shanahan F, Quigley EM, Fitzgerald GF, Ross RP. 644 2011. Recombinant lactobacilli expressing linoleic acid isomerase can modulate the fatty acid 645 composition of host adipose tissue in mice. Microbiology 157:609-615. 
646 Sadrzadeh-Yeganeh H, Elmadfa I, Djazayery A, Jalali M, Heshmat R, Chamary M. 2010. The effects of 647 probiotic and conventional yoghurt on lipid profile in women. British Journal of Nutrition $648 \quad 103: 1778-1783$.

649 Salonen A, Nikkilä J, Jalanka-Tuovinen J, Immonen O, Rajilić-Stojanović M, Kekkonen RA, Palva A, 650 de Vos WM. 2010. Comparative analysis of fecal DNA extraction methods with phylogenetic

651 microarray: effective recovery of bacterial and archaeal DNA using mechanical cell lysis. Journal of 652 Microbiological Methods 81:127-134.

653 Satokari RM, Vaughan EE, Akkermans AD, Saarela M, de Vos WM. 2001. Polymerase chain reaction 654 and denaturing gradient gel electrophoresis monitoring of fecal bifidobacterium populations in a 655 prebiotic and probiotic feeding trial. Systematic and Applied Microbiology 24:227-231.

656 Savard P, Lamarche B, Paradis ME, Thiboutot H, Laurin É, Roy D. 2011. Impact of Bifidobacterium 657 animalis subsp. lactis BB-12 and Lactobacillus acidophilus LA-5-containing yoghurt on fecal bacterial 658 counts of healthy adults. International Journal of Food Microbiology 149:50-57.

659 Saxelin M, Tynkkynen S, Mattila-Sandholm T, de Vos WM. 2005. Probiotic and other functional 660 microbes: from markets to mechanisms. Current Opinion in Biotechnology 16:204-211.

661 Shen HJ, Huang JZ. 2008. Sparse principal component analysis via regularized low rank matrix 662 approximation. Journal of Multivariate Analysis 99:1015-1034.

663 Sekirov I. 2010. Gut microbiota in health and disease. Physiological Reviews 90:859-904.

664 Smyth GK. 2004. Linear models and empirical Bayes methods for assessing differential expression in 665 microarray experiments. Statistical Applications in Genetics and Molecular Biology 3:Article 3.

666 Storey JD, Tibshirani R. 2003. Statistical significance for genomewide studies. Proceedings of the 667 National Academy of Sciences U S A 100:9440-9445. 
668 Swann JR, Want EJ, Geier FM, Spagou K, Wilson ID, Sidaway JE, Nicholson JK, Holmes E. 2011.

669 Systemic gut microbial modulation of bile acid metabolism in host tissue compartments. Proceedings 670 of the National Academy of Sciences U S A 108 Suppl 1:4523-4530.

671 Tremaroli V, Bäckhed F. 2012. Functional interactions between the gut microbiota and host 672 metabolism. Nature 489:242-249.

673 Vaughan EE, Mollet B, de Vos WM. 1999. Functionality of probiotics and intestinal lactobacilli: light 674 in the intestinal tract tunnel. Current Opinion in Biotechnology 10:505-510.

675 Velagapudi VR, Hezaveh R, Reigstad CS, Gopalacharyulu P, Yetukuri L, Islam S, Felin J, Perkins R, 676 Borén J, Orešič M, Bäckhed F. 2010. The gut microbiota modulates host energy and lipid metabolism 677 in mice. Journal of Lipid Research 51:1101-1112.

678 Vitali B, Ndagijimana M, Cruciani F, Carnevali P, Candela M, Guerzoni ME, Brigidi P. 2010. Impact of 679 a synbiotic food on the gut microbial ecology and metabolic profiles. BMC Microbiology 10:4.

680 Wallner S, Schmitz G. 2011. Plasmalogens the neglected regulatory and scavenging lipid species.

681 Chemistry and Physics of Lipids 164:573-589.

682 Wang Z, Klipfell E, Bennett BJ, Koeth R, Levison BS, DuGar B, Feldstein AE, Britt EB, Fu X, Chung 683 Y-M, Wu Y, Schauer P, Smith JD, Allayee H, Tang WHW, DiDonato JA, Lusis AJ, Hazen SL. 2011. 684 Gut flora metabolism of phosphatidylcholine promotes cardiovascular disease. Nature 472:57-63.

685 Westerbacka J, Kotronen A, Fielding BA, Wahren J, Hodson L, Perttilä J, Seppänen-Laakso T, Suortti 686 T, Arola J, Hultcrantz R, Castillo S, Olkkonen VM, Frayn KN, Orešič M, Yki-Järvinen H. 2010. 687 Splanchnic balance of free fatty acids, endocannabinoids, and lipids in subjects with nonalcoholic fatty 688 liver disease. Gastroenterology 139:1961-1971. 
689 Yamanoa T, Iinoa H, Takadaa M, Bluma S, Rochata F, Fukushima Y. 2006. Improvement of the human 690 intestinal flora by ingestion of the probiotic strain Lactobacillus johnsonii La1. British Journal of 691 Nutrition 95:303-312.

692 Zoetendal EG, Rajilić-Stojanović M, de Vos WM. 2008. High-throughput diversity and functionality 693 analysis of the gastrointestinal tract microbiota. Gut 57:1605-1615. 


\section{Table $\mathbf{1}_{\text {(on next page) }}$}

Stability of microbiota and lipid profiles in the probiotic and placebo groups

We determined the similarity (expressed as Pearson's correlation) both within and between the time points (TP) for the microbiota and lipid profiles by the average scatter $r$ of the profiles. Lipid data is available for the first two time points (TP1 and TP2, three weeks before the intervention and during the intervention , respectively), and not available (-) for the third time point (TP3) measured three weeks after the intervention. 
Between subjects

Microbiota

Probiotic

Placebo

Lipids

Probiotic

Placebo
TP2

0.78

0.77

0.89

0.89
Within subjects

TP3

0.78

0.77

TP1 vs TP2

TP3 vs TP2

0.94

0.95

0.94

0.95

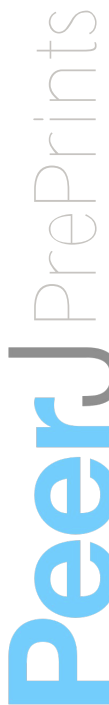




\section{Table $2_{\text {(on next page) }}$}

Associations between genus-level bacterial groups and enzymatically determined lipids

Associations between the relative amounts of genus-level bacterial groups as determined by the HITChip analysis and the serum lipid concentrations are quantified with a biweight correlation. Only significant positive and negative correlations are shown ( $(\mathbf{q}<0.05$; otherwise '-'). Abbreviations: Total cholesterol (TC), high-density lipoprotein (HDL) and low-density lipoprotein (LDL) cholesterol, triglyceride (TG). Correlations between genus-level bacterial groups and mass spectrometry-determined lipids are provided in Supplementary Table S3. 


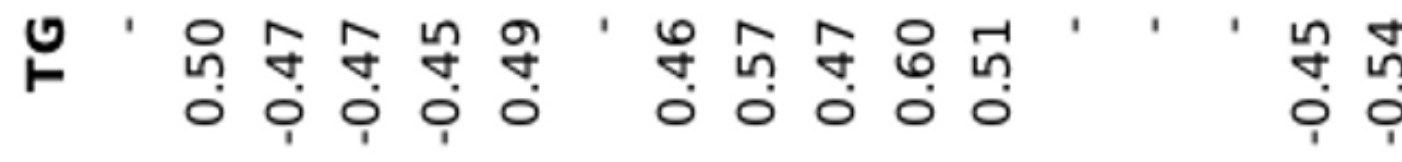

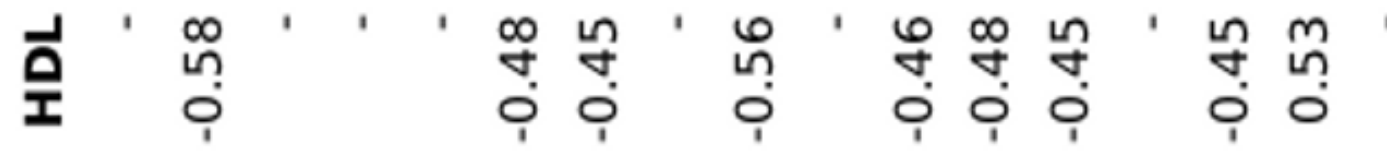

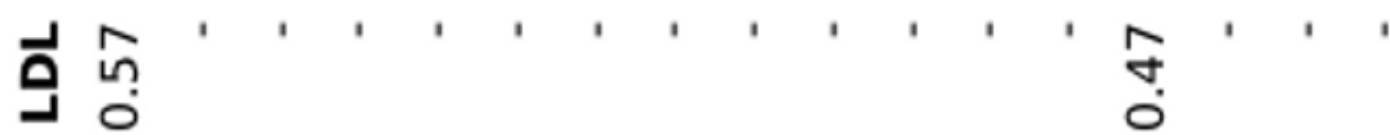

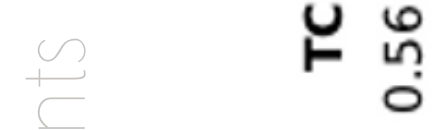

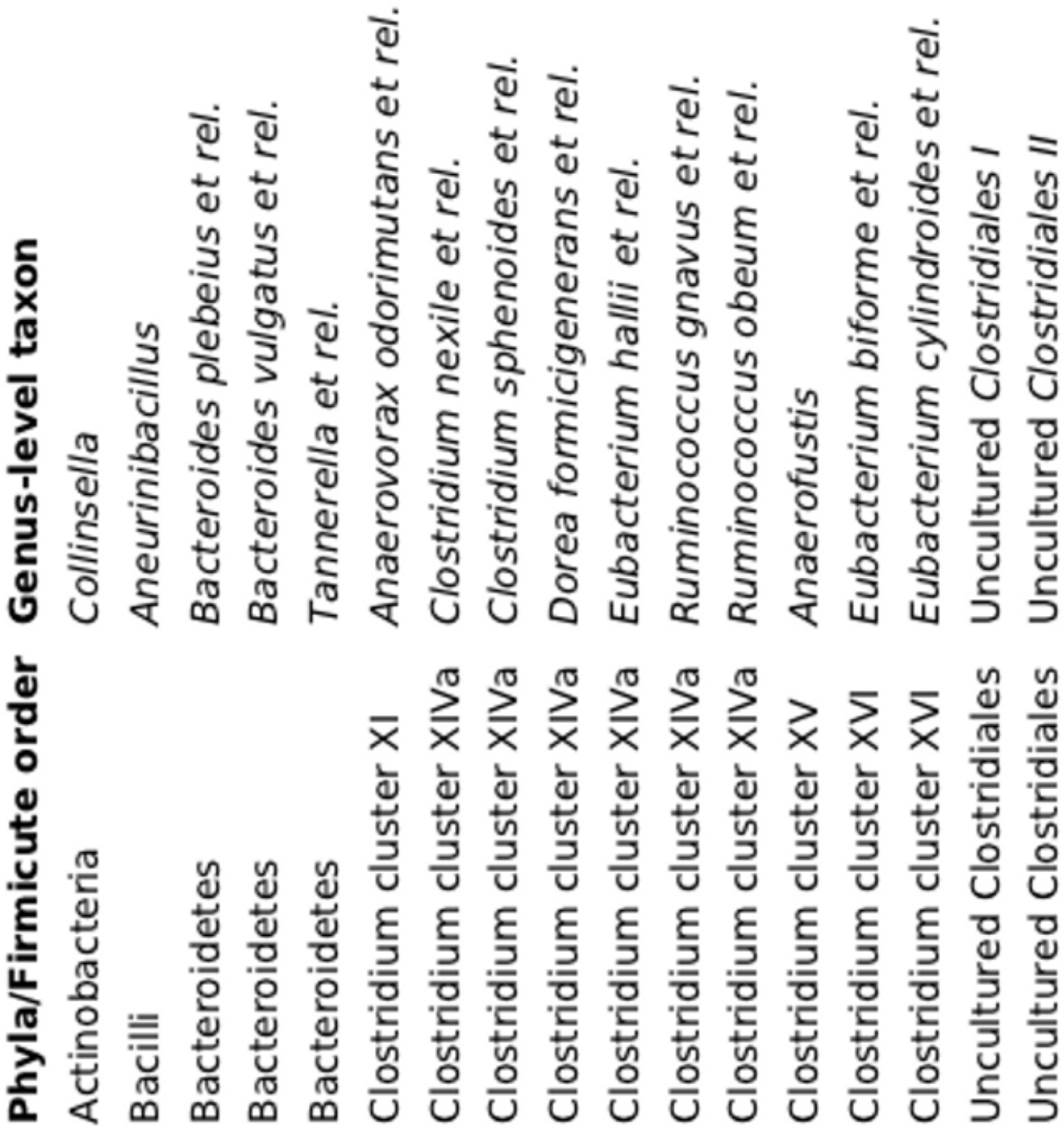




\section{Figure 1}

Intervention effects on the abundance of $L$. rhamnosus.

Mean abundance of L. rhamnosus among the study subjects before, during and after the probiotic intervention (the time points 1-3, respectively) quantified by the HITChip hybridization signal. The error bars denote the Gaussian 95\% confidence limits based on standard deviation of the mean.

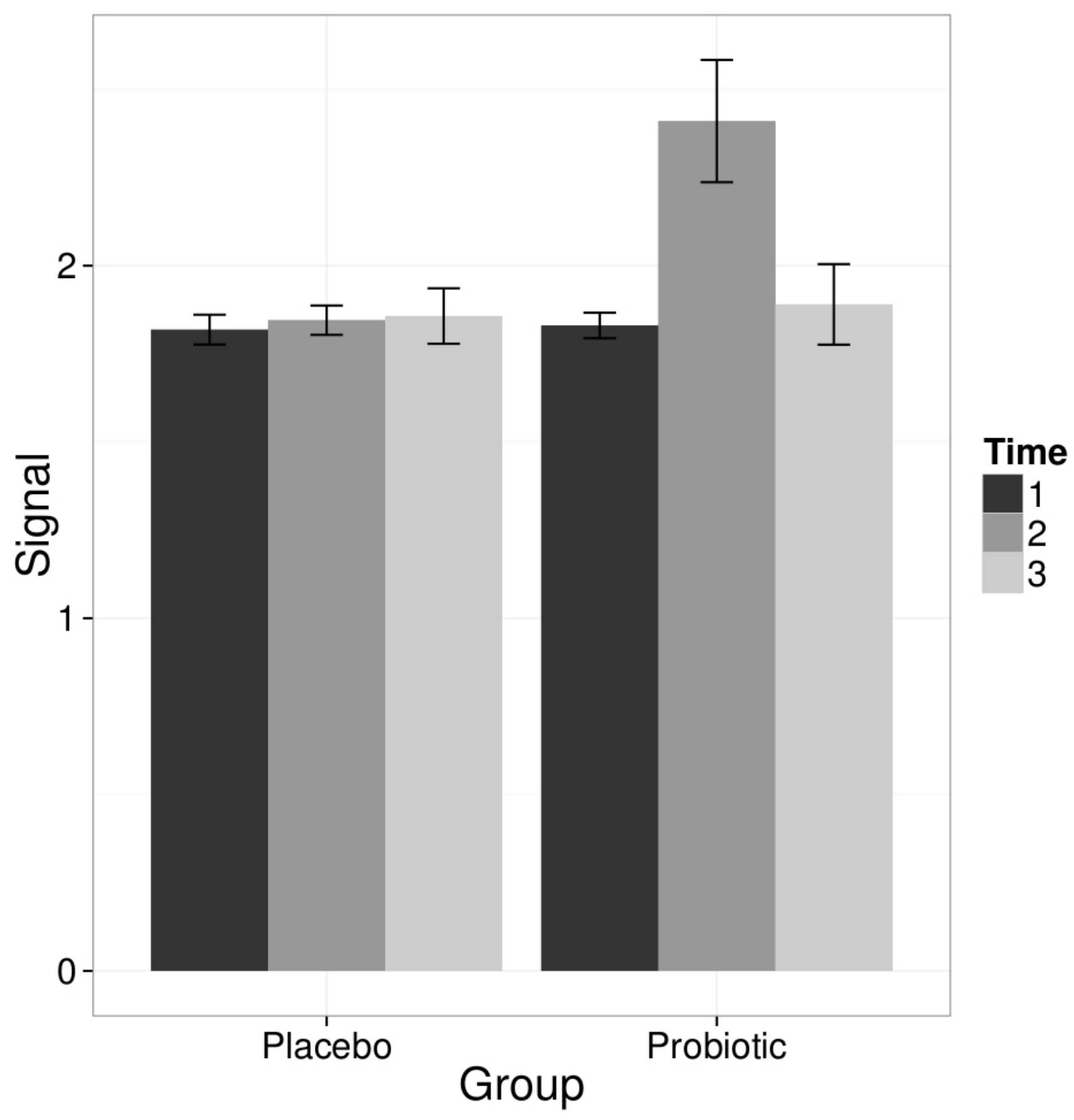




\section{Figure 2}

Correlations between intestinal genus-level phylogenetic groups and serum lipids

The correlations between the intestinal bacteria and serum lipids are indicated by colors (red: positive; blue: negative). The significant correlations $(q<0.05)$ are indicated by '+'; only lipids and bacteria with at least one significant correlation are shown. Hierarchical clustering of the rows and columns highlights groups of significantly correlated bacteria and lipids. Lipids have been named according to Lipid Maps (http://www.lipidmaps.org ) with the following abbreviations: Cer: ceramide; ChoE: cholesteryl ester; lysoPC: lysophosphatidylcholine; PA: phosphatidic acid; PG: phosphatidylglycerol; PC: phosphatidylcholine; PS: phosphatidylserine; SM: sphingomyelin; TG: triglyceride. Where the fatty acid composition could not be determined, the total number of carbons and double bonds is indicated. The first number indicates the amount of carbon atoms in the fatty acid molecule, followed by the number of double bonds. For further details, see the Methods section.

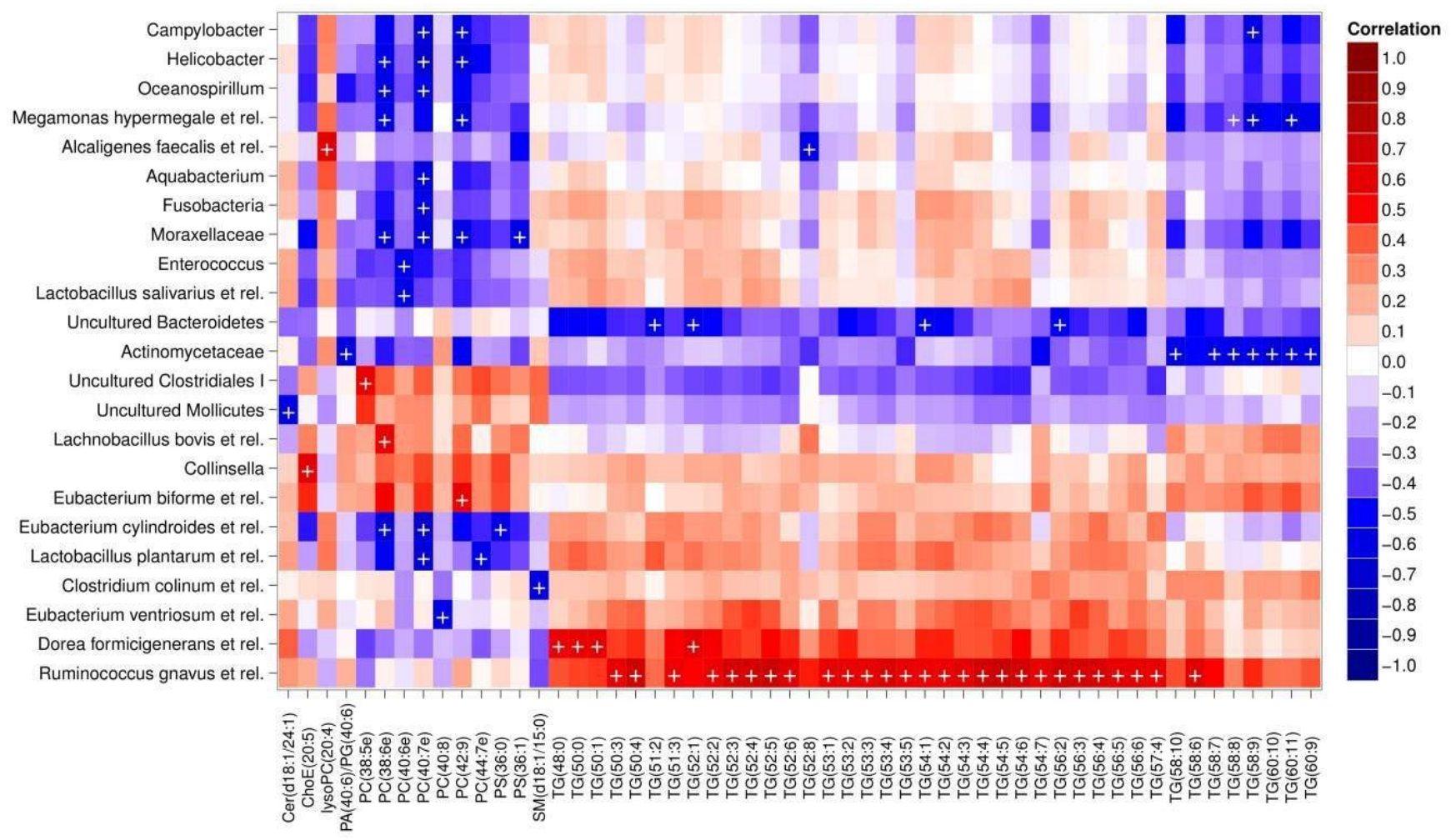




\section{Figure 3}

Association between Ruminococcus gnavus et rel. and serum triglyceride (TG) lipids

The relative amounts of $R$. gnavus et rel. were quantified by the HITChip analysis and the triglyceride concentration was determined based on two independent techniques: A the triglyceride TG(54:5) (see Fig. 2 for explanation) by mass spectrometry ( $\mathrm{r}=0.61)$; $\mathbf{B}$ triglyceride by an enzymatic assay ( $\mathrm{r}=0.60)$.
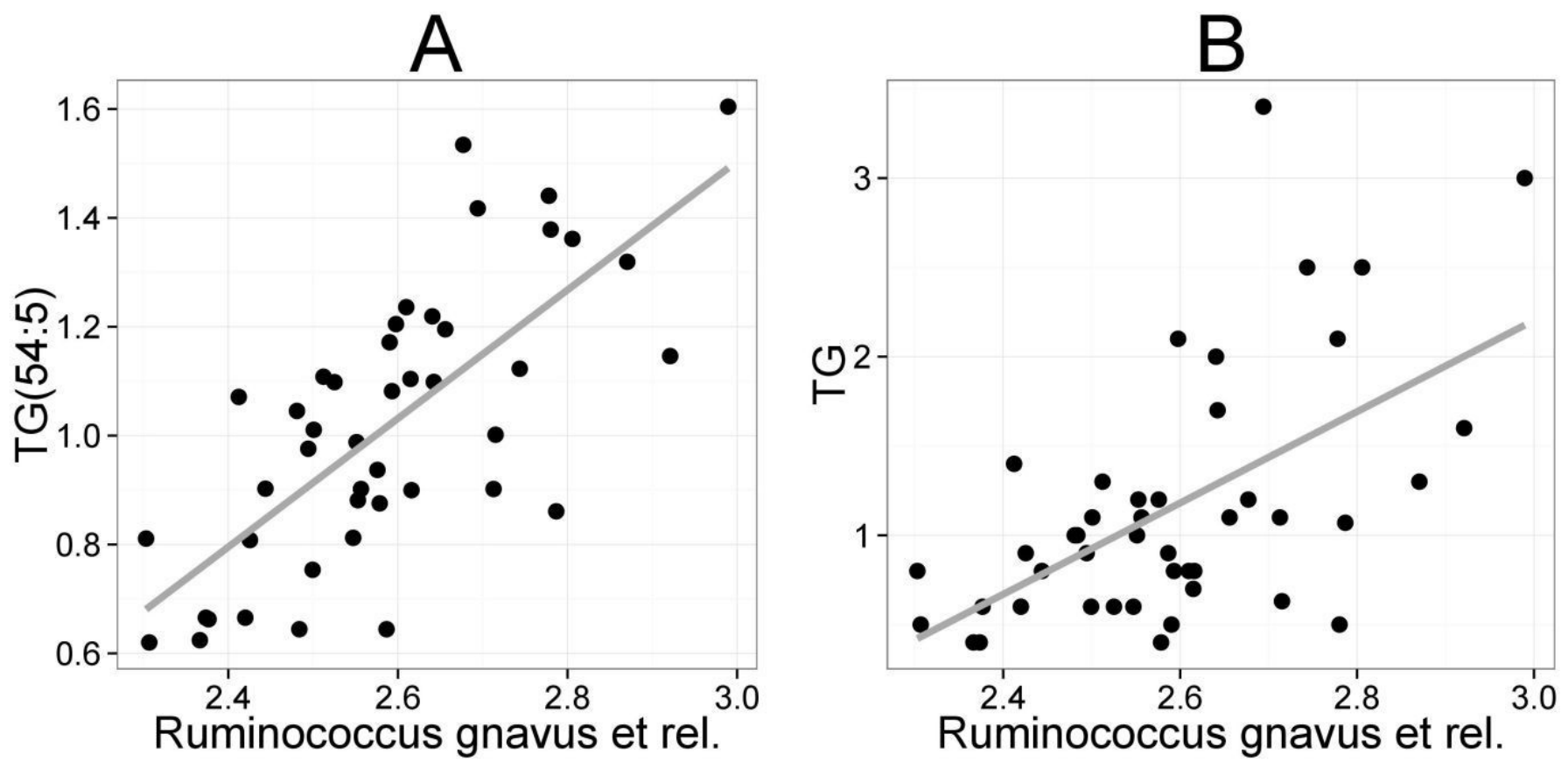


\section{Figure 4}

Association between Collinsella spp. and serum cholesterol

The relative amounts of Collinsella spp. were quantified by the HITChip analysis, and serum cholesterol levels were determined by two independent techniques: A Cholesterol ester $\operatorname{ChoE(20:5)}$ (see Fig. 2 for explanation) by mass spectrometry (r=0.59); B low-density lipoprotein (LDL) cholesterol by enzymatic assay ( $\mathrm{r}=0.57)$.
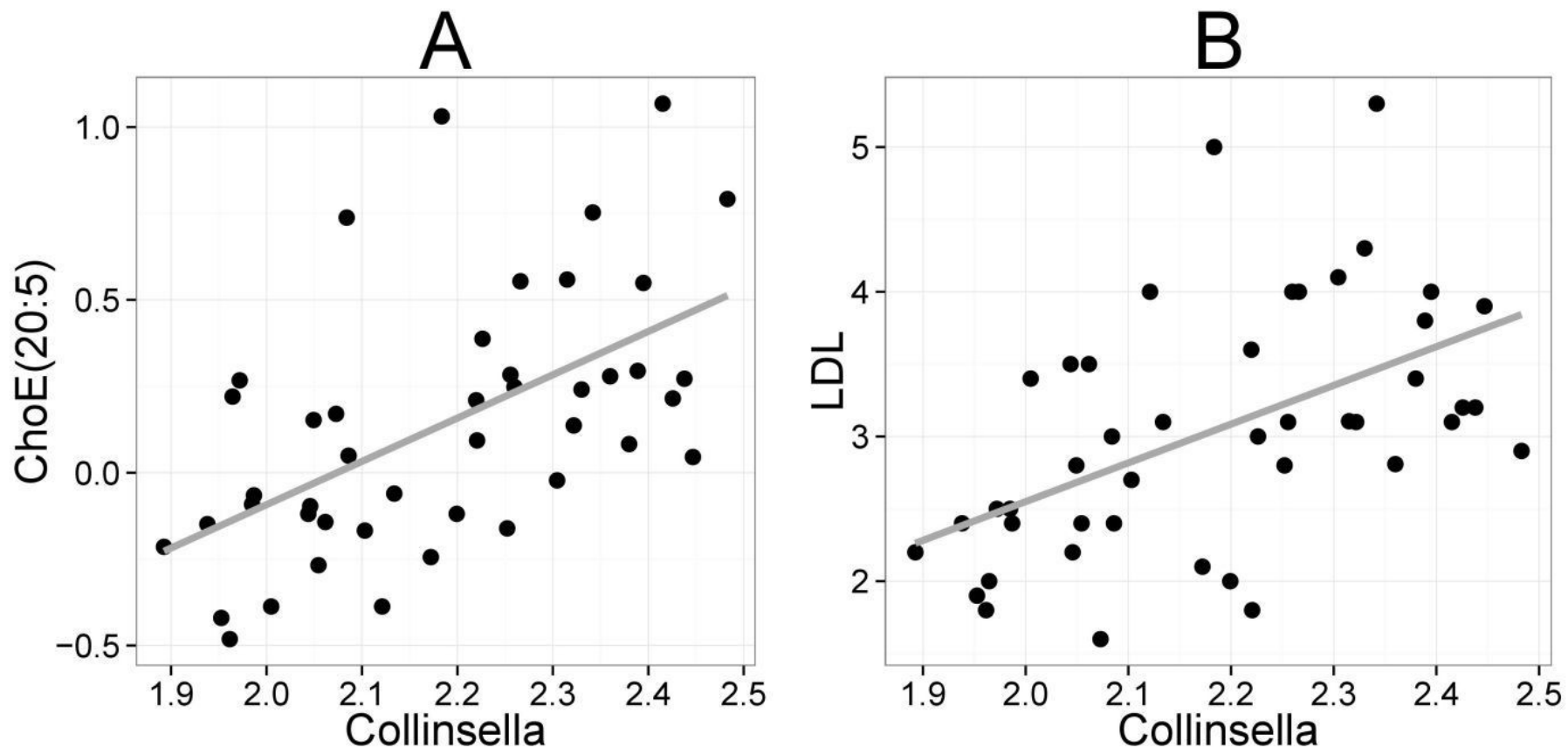